\title{
IMPACTO DA INTERVENÇÃO DO ENFERMEIRO DE REABILITAÇÃO À PESSOA COM DOENÇA PULMONAR OBSTRUTIVA CRÓNICA - REVISÃO SISTEMÁTICA
}

\section{IMPACTO DE LA INTERVENCIÓN DEL ENFERMERO DE REHABILITACIÓN A LA PERSONA CON ENFERMEDAD PULMONAR OBSTRUCTIVA CRÓNICA - REVISIÓN SISTEMÁTICA}

\section{IMPACT OF THE REHABILITATION NURSE'S INTERVENTION ON THE PERSON WITH CHRONIC OBSTRUCTIVE PULMONARY DISEASE - SYSTEMATIC REVIEW}

DOI 10.33194/rper.2019.v2.n2.02.4572 | Submetido 08.05.2019 | Aprovado 03.12.2019

\author{
Sara Varão ${ }^{1}$; ; Cristina Saraiva² \\ 1 - Centro Hospitalar Universitário Lisboa Central; 2 - Escola Superior de Enfermagem de Lisboa
}

\section{RESUMO}

Introdução: A Doença Pulmonar Obstrutiva Crónica é prevenível e tratável, com manifestações respiratórias persistentes, nas quais as intervenções do ER podem contribuir para melhorar a capacidade funcional e emocional, o conhecimento, promover o autocuidado, a adesão ao regime terapêutico e a comportamentos saudáveis.

Objetivo: Sintetizar evidência científica sobre impacto da intervenção do ER na Pessoa com DPOC.

Métodos: Estabeleceu-se como pergunta de investigação "Qual o impacto da intervenção do Enfermeiro de Reabilitação na Pessoa com Doença Pulmonar Obstrutiva Crónica?”, adotando-se a metodologia do The Joanna Briggs Institute para revisão sistemática. Definiram-se critérios de inclusão e a pesquisa booleana, na EBSCOhost.

Resultados: Incluíram-se 6 artigos, que retratam a componente educacional e suporte do ER. O ER tem impacto positivo na vida da pessoa com DPOC, verificando-se melhores resultados no controlo sintomático/dispneia, na redução das admissões e tempo de internamento, no aumento da perceção da doença e da qualidade de vida. Tem impacto na melhoria das atividades de vida diária e na redução dos custos.

Conclusões: Os estudos evidenciaram variedade nas formas de atuação do ER, mas impacto positivo associado. Salienta-se pouca investigação sobre resultados das intervenções.

Descritores: Doença Pulmonar Obstrutiva Crónica. Enfermagem de Reabilitação. Reabilitação Respiratória

\section{RESUMEN}

Introducción: la enfermedad pulmonar obstructiva crónica es prevenible y tratable, con manifestaciones respiratorias y limitación del flujo de aire, en la cual las intervenciones de ER pueden contribuir a mejorar la capacidad funcional y emocional, el conocimiento, promover el autocuidado, el cumplimiento del régimen terapéutico y los comportamientos saludables.

Objetivo General: Sintetizar la evidencia cientifica sobre el impacto del ER en la Persona con EPOC.

Métodos: Se estableció como pregunta de investigación "¿Cuál es el impacto de la intervención del Enfermero de Rehabilitación en la persona con con Enfermedad Pulmonar Obstructiva Crónica?", Se adoptó la metodología del Instituto Joanna Briggs para la revisión sistemática. Se definieron criterios de inclusión y la como investigación booleana, en la base EBSCOhost.

Resultados: Incluimos 6 artículos, que retratan e el componente educativo y de apoyo de la ER. El ER tiene un impacto positivo en la vida de la persona com EPOC, com mejores resultados en términos de control sintomático/disnea, en la reducción de las admisiones y tiempo de internamiento, en el aumento de la percepción del control de la enfermedad y de la calidad de vida. Tiene impacto en la mejora de las actividades de vida diaria y en la reducción de los costos.

Conclusiones: Los estudios evidenciaron variedad en las formas de actuación del ER, pero impacto positivo. Se destaca la escasa investigación sobre los resultados de las intervenciones.

Palabras clave: Enfermedad Pulmonar Obstructiva Crónica. Enfermería en Rehabilitación. Rehabilitación Respiratoria

\section{ABSTRACT}

Introdution: Chronic Obstructive Pulmonary Disease is preventable and treatable, with respiratory manifestations and airflow limitation, in which Intervention can contribute to improve functional and emotional capacity, knowledge, promote self-care, adherence to the therapeutic regimen and healthy behaviors.

General Objective: To synthesize scientific evidence of the impact of the RN's intervention on the Person with COPD. 
Methods: It was established as a research question "What is the impact of the Rehabilitation Nurse intervention on a person with Chronic Obstructive Pulmonary Disease?". It were defined inclusion criteria and it was defined a boolean research, in the EBSCOhost database. It was adopted the methodology oh the Joanna Briggs Institute for the systematic review.

Results: We included 6 articles, which mainly portrayed the educational and support component of the RN. The RN has a positive impact on the life of the person with COPD, with better results in terms of symptomatic control/dyspnea, on the reduction of admissions and length of hospitalization, increased awareness of disease control and quality of life. It has an impact on improving daily life activities and reducing costs.

Conclusions: The studies evidenced variety in the forms of performance of the RN, but positive impact. There is little research on the results of interventions.

Keywords: Pulmonary Disease, Chronic Obstructive. Rehabilitation Nursing. Respiratory Rehabilitation

\section{INTRODUÇÃO}

A Doença Pulmonar Obstrutiva Crónica (DPOC) é uma doença prevenível e tratável, que se caracteriza por manifestações respiratórias persistentes e com limitação do fluxo aéreo, devido a exposição significativa de partículas/gases nocivos (1). A DPOC é responsável por despesas elevadas para a sociedade, tendo um considerável impacto económico, que é determinado a partir dos custos diretos (diagnóstico e tratamento) e os custos indiretos (consequências das incapacidades provocadas pela DPOC como as perdas decorrentes da invalidez, do absenteísmo, da mortalidade prematura e do pagamento de benefícios) (2). É um facto que Portugal integra o grupo de países com menor mortalidade por DPOC, com uma taxa de $8,0 \%$ (630 pessoas) ${ }^{(3)}$. Porém, em 2016, a DPOC foi responsável por 7864 internamentos, estando presente em 1963 pessoas submetidas a ventilação não invasiva. Em 2016, correspondeu a 7,0\% dos internamentos por causa respiratória, sendo ultrapassada pelos internamentos por pneumonias e por insuficiência respiratória. Tal traduz-se num considerável peso social e económico para o país, tal como referido. Por outras palavras, traduz-se em consideráveis custos diretos e indiretos com (re)internamentos e complicações, que poderiam ser evitáveis com recurso à reabilitação das pessoas com DPOC. Por esta razão, em Portugal, até 2020, pretende-se reduzir em 10\% (face a 2014), os internamentos devido a causas respiratórias (das quais a DPOC), que possam ser prevenidos ou tratados em centros de saúde ${ }^{(4)}$.

Uma das estratégias é a implementação de programas de reabilitação respiratória, isto é, a adoção de uma intervenção baseada na avaliação da pessoa, seguida de terapias personalizadas que englobem treino e exercício, educação, mudança de comportamento e adesão/gestão de comportamentos promotores de bem-estar ${ }^{(1)}$. Tal ganha sentido, quando se percebe que esta traz benefícios às pessoas com DPOC, tendo sido demonstrado que é a estratégia terapêutica mais efetiva em pessoas estáveis, sendo responsável por vários ganhos como: redução de hospitalizações (entre pessoas que tiveram recentes exacerbações), melhoria da dispneia, melhoria da tolerância ao exercício, controlo sintomático ou melhoria da qualidade de vida (1). Na mesma ótica, a nível nacional, foi clarificada a necessidade e importância da Reabilitação Respiratória na pessoa com DPOC, salientando-se os ganhos passíveis de ser obtidos, como a melhoria da dispneia e qualidade, bem como a diminuição de dias de hospitalização ${ }^{{ }^{(5)}}$. A reabilitação respiratória deve ser encarada como uma intervenção global e multidisciplinar, devendo ser individualizada e englobando treino ao esforço, intervenção psicossocial, com vista à redução de sintomas, otimização da funcionalidade, aumento da participação social e redução de custos. Desta forma, a equipa de reabilitação deve ser composta por pneumologista, fisiatra, fisioterapeuta, psicólogo, nutricionista, assistente social e, inevitavelmente, pelo Enfermeiro de Reabilitação ${ }^{(5)}$.

Face ao descrito, torna-se essencial a intervenção do Enfermeiro de Reabilitação no processo de reabilitação da pessoa com DPOC. De facto, está descrito que o Enfermeiro de Reabilitação deve atuar ao nível da otimização terapêutica (verificando e corrigindo a técnica inalatória e o dispositivo inalatório mais adequado), da educação da pessoa/família e da reeducação funcional respiratória e do treino e exercício ${ }^{(6)}$. Relativamente à componente educacional, - Enfermeiro de Reabilitação deve incidir nas necessidades da pessoa/família, procurando desmistificar a DPOC e incidindo na necessidade da prática de exercício, da cessação tabágica, da adoção de técnicas de conservação de energia ou nos aspetos relacionados com a alimentação. Por sua vez, a reeducação funcional respiratória deve ser uma componente do programa de reabilitação respiratória do Enfermeiro de Reabilitação, englobando exercícios respiratórios, a consciencialização dos tempos respiratórios, a (re)aprendizagem da respiração diafragmática e a expiração com lábios semicerrados. Da mesma forma, o treino dos músculos respiratórios, dos membros superiores e inferiores deve ser uma preocupação do Enfermeiro de Reabilitação ${ }^{(6)}$.

Perante o descrito, o Colégio da Especialidade de Enfermagem de Reabilitação identificou como uma das áreas de investigação prioritárias a curto prazo, o estudo sobre o impacto das intervenções do Enfermeiro de Reabilitação ${ }^{(7)}$.

Face ao descrito, torna-se importante compreender qual o efeito da intervenção do Enfermeiro de Reabilitação na pessoa com DPOC e que ganhos daí advêm. Assim, torna-se importante elaborar uma revisão sistemática da literatura que vise atingir o seguinte objetivo: sintetizar a evidência sobre 0 impacto que a intervenção do Enfermeiro de Reabilitação tem na Pessoa com DPOC. Para tal, 
definiu-se como ponto de partida a seguinte questão de pesquisa: Qual o impacto da intervenção do Enfermeiro de Reabilitação na Pessoa com DPOC?

\section{MÉTODO}

O presente manuscrito trata-se de uma revisão sistemática da literatura. Para o seu propósito, adotouse como referencial metodológico as etapas e recomendações do Joanna Briggs Institute ${ }^{(8)}$. Para a construção da questão de pesquisa e para a identificação dos conceitos-chave recorreu-se ao método PICO (Population, Intervention, Comparision e Outcomes) ${ }^{(8)}$, como se pode ver na Quadro 1.

\begin{tabular}{|c|c|c|c|}
\hline $\mathbf{P}$ & População & $\begin{array}{l}\text { Pessoa com Doença } \\
\text { Pulmonar Obstrutiva } \\
\text { Crónica }\end{array}$ & $\begin{array}{l}\text { Conceitos-chave: } \\
\text { - Doença pulmonar } \\
\text { obstrutiva }\end{array}$ \\
\hline I & Intervenção & $\begin{array}{l}\text { Intervenção do } \\
\text { Enfermeiro de } \\
\text { Reabilitação }\end{array}$ & $\begin{array}{l}\text { crónica/chronic } \\
\text { obstructive } \\
\text { pulmonar disease }\end{array}$ \\
\hline C & Comparação & Se existente & 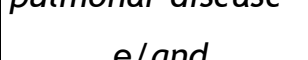 \\
\hline 0 & Resultados & $\begin{array}{l}\text { Decorrentes da } \\
\text { intervenção do } \\
\text { Enfermeiro de } \\
\text { Reabilitação em } \\
\text { qualquer contexto }\end{array}$ & $\begin{array}{l}\text { - Impacto/Impact } \\
\text { e/and } \\
\text {-Enfermeiro/Nurse }\end{array}$ \\
\hline
\end{tabular}

Fonte: Adaptado pelas autoras com base em The Joanna Briggs Institute (2011)

Quadro 1 - Identificação dos conceitos-chave através da mnemónica PICO

Com o método PICO definiram-se critérios de inclusão e exclusão, elencados no quadro 2.

Importa elencar que a escolha do período de publicação (últimos 5 anos) prendeu-se com o facto da atualização temporal das referências ser um dos pontos cruciais do rigor científico da mesma, pretendendo-se sintetizar a evidência mais atual sobre o impacto do Enfermeiro de reabilitação. Paralelamente, este limite justifica-se pelo facto da preocupação pelo impacto do enfermeiro de reabilitação ter surgido nos últimos anos.

Face ao propósito da revisão sistemática, definiu-se como expressão booleana (impact) AND (nurse) AND (chronic obstructive pulmonary disease). Em abril de 2018, procedeu-se à pesquisa eletrónica na base de dados EBSCOhost (na CINAHL Plus, na MEDLINE, Academic Search Complete, Business Source Complete, MedicaLatina, Chrocrane Central Register of Controlled Trials), obtendo-se 250 artigos. Selecionou-se a opção 'Full Text' (132 artigos), dentro do limite temporal 2013-2018, em inglês (não havia artigos disponíveis em português), ficando 62 artigos. Destes, foram eliminados (pela própria base de dados) os artigos duplicados, obtendo-se 39 artigos. Paralelamente, fezse nova pesquisa na PubMed, com a mesma pesquisa booleana (118 artigos). Selecionou-se 'Full text' (111 artigos) e o limite temporal dos últimos 5 anos, ficando com 44 artigos. Também na PubMed não havia produção de artigos em português.

\begin{tabular}{|c|c|c|}
\hline $\begin{array}{l}\text { Critérios de } \\
\text { Seleção }\end{array}$ & $\begin{array}{l}\text { Critérios de } \\
\text { Inclusão }\end{array}$ & $\begin{array}{c}\text { Critérios de } \\
\text { Exclusão }\end{array}$ \\
\hline $\begin{array}{c}\text { Tipo de } \\
\text { Participantes }\end{array}$ & $\begin{array}{c}\text { Pessoa com } \\
\text { Doença Pulmonar } \\
\text { Obstrutiva Crónica }\end{array}$ & $\begin{array}{l}\text { Pessoa com outro } \\
\text { tipo de patologia }\end{array}$ \\
\hline $\begin{array}{c}\text { Tipo de } \\
\text { Intervenção }\end{array}$ & $\begin{array}{l}\text { Intervenção do } \\
\text { Enfermeiro de } \\
\text { Reabilitação }\end{array}$ & $\begin{array}{c}\text { O que não se refira } \\
\text { unicamente à } \\
\text { intervenção do } \\
\text { Enfermeiro } \\
\text { Reabilitação }\end{array}$ \\
\hline $\begin{array}{l}\text { Tipo de } \\
\text { resultados }\end{array}$ & $\begin{array}{c}\text { Decorrentes da } \\
\text { intervenção em } \\
\text { qualquer contexto }\end{array}$ & $---\cdot$ \\
\hline $\begin{array}{l}\text { Tipo de } \\
\text { Estudos }\end{array}$ & $\begin{array}{c}\text { Estudos de } \\
\text { investigação } \\
\text { quantitativos ou } \\
\text { qualitativos }\end{array}$ & $\begin{array}{l}\text { Estudos mistos, não } \\
\text { científicos e sem } \\
\text { resumo na base de } \\
\text { dados }\end{array}$ \\
\hline $\begin{array}{c}\text { Data de } \\
\text { Publicação }\end{array}$ & Entre 2013-2018 & Antes de 2013 \\
\hline Idioma & $\begin{array}{l}\text { Inglês ou } \\
\text { português }\end{array}$ & $\begin{array}{l}\text { Outro idioma que } \\
\text { não inglês ou } \\
\text { português }\end{array}$ \\
\hline
\end{tabular}

Quadro 2. Definição dos critérios de Inclusão e Exclusão

Dos 83 artigos das 2 bases de dados, eliminaram-se as referências duplicadas (20), ficando 63 artigos. Eliminaram-se os artigos que não apresentavam texto completo ou exigiam subscrição ${ }^{(17)}$, ficando com 46 artigos. Seguiu-se a leitura do título e resumo, de modo a verificar o cumprimento dos critérios de inclusão. Por não cumprirem os critérios, foram excluídos 40 artigos, sendo que 6 artigos constituíram o corpus de análise, como se pode observar na Figura 1.
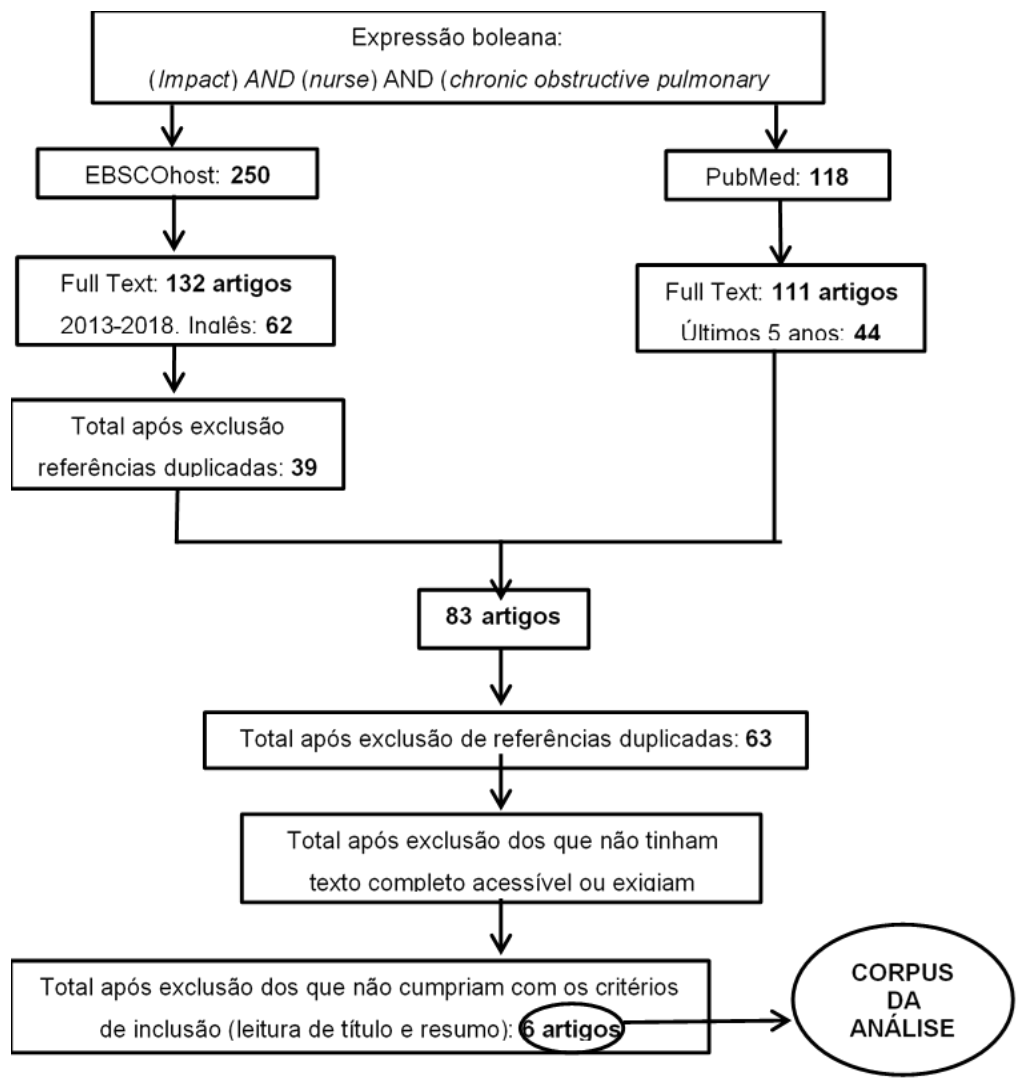

Fluxograma 1 - Identificação do Corpus de Análise 


\section{Avaliação Da Qualidade Metodológica}

Após a obtenção de 6 estudos, constituintes do corpus da análise, seguiu-se à sua avaliação da qualidade metodológica. Para a avaliação de 4 artigos recorreu-se ao instrumento Meta Analysis of Statistics Assessment and Review Instrument critical appraisal tools Randomized Control/Pseudo-Randomized Trial, com 10 critérios ${ }^{(8)}$. Para a avaliação dos outros 2 artigos, recorreu-se ao instrumento Meta Analysis of Statistics
Assessment and Review Instrument critical appraisal tools Descriptive/Case Series Studies com 9 critérios ${ }^{(6)}$.

O Quadro 3 demonstra que foram incluídos todos os estudos com pontuação igual ou superior a $70 \%$ dos critérios (pontuação igual ou superior a 7), mantendose um corpus da análise de 6 artigos. O Quadro 3 demonstra, ainda, a avaliação dos níveis de evidência de cada artigo, de acordo com Joanna Briggs Institute Levels of Evidence( ${ }^{(8)}$.

\begin{tabular}{|c|c|c|c|}
\hline Estudos & Instrumento Utilizado & Pontuação & $\begin{array}{c}\text { Nível de } \\
\text { Evidência }\end{array}$ \\
\hline $\begin{array}{l}\text { Champion, R., Hall, T., \& Tori, K. (2015). Home oxygen therapy } \\
\text { assessment for COPD patients discharged from hospital: } \\
\text { Respiratory NP Model of Care. }\end{array}$ & \multirow{4}{*}{$\begin{array}{l}\text { Meta Analysis of } \\
\text { Statistics Assessment } \\
\text { and Review } \\
\text { Instrument critical } \\
\text { appraisal tools } \\
\text { Randomized } \\
\text { Control/Pseudo- } \\
\text { Randomized Trial }\end{array}$} & $8 / 10$ & E2 \\
\hline $\begin{array}{l}\text { Kim, J., Park, E.-C., \& Han, K.-T. (2016). Nurse Staffing and 30-day } \\
\text { Readmission of Chronic Obstructive Pulmonary Disease Patients: A } \\
\text { 10-year Retrospective Study of Patient Hospitalization. }\end{array}$ & & $7 / 10$ & E2 \\
\hline $\begin{array}{l}\text { Bilington, J., Coster, S., Murrells, T., \& Norman, I. (2015). } \\
\text { Evaluation of a Nurse-Led Educational Telephone Intervention to } \\
\text { Support Self-Management of Patients With Chronic Obstructive } \\
\text { Pulmonary Disease: A Randomized Feasibility Study. }\end{array}$ & & $10 / 10$ & F1 \\
\hline $\begin{array}{l}\text { Weldam, S. W., Schuurmans, M. J., Zanen, P., Heijmans, M. J., } \\
\text { Sachs, A. P., \& Lammers, J.-W. J. (2017). The effectiveness of a } \\
\text { nurse-led illness perception intervention in COPD patients: a } \\
\text { cluster randomised trial in primary care. }\end{array}$ & & $9 / 10$ & E1 \\
\hline $\begin{array}{l}\text { Duangubpha, S., Hanucharurnkul, S., Pookboonmee, R., Orathai, } \\
\text { P., \& Kiatboonri, C. (April-June de 2013). Chronic Care Model } \\
\text { Implementation and Outcomes among Patients with COPD in Care } \\
\text { Teams with and without Advanced Practice Nurses. }\end{array}$ & \multirow{2}{*}{$\begin{array}{l}\text { Meta Analysis of } \\
\text { Statistics Assessment } \\
\text { and Review } \\
\text { Instrument critical } \\
\text { appraisal tools } \\
\text { Descriptive/Case } \\
\text { Series Studies }\end{array}$} & $7 / 9$ & $\mathrm{E} 1$ \\
\hline $\begin{array}{l}\text { Cox, K., Macleod, S. C., Sim, C. J., Jones, A. W., \& Trueman, J. } \\
\text { (2017). Avoiding hospital admission in COPD impact of a specialist } \\
\text { nursing team. }\end{array}$ & & $7 / 9$ & E2 \\
\hline
\end{tabular}

Quadro 3. Avaliação da Qualidade Metodológica e do Nível de Evidência dos Estudos

\section{RESULTADOS}

Após a avaliação metodológica, procedeu-se à extração e síntese de dados.

Alguns autores elaboraram um estudo que tinha como objetivo avaliar a efetividade da intervenção do enfermeiro (Respiratory Nurse), em contexto de cuidados de saúde primários (9). Para isso, implementaram um tipo de intervenção, a Chronic Obstructive Pulmonary Disease- Guidance, Research on Illness Perception (COPD-GRIP). Os autores realizaram um ensaio controlado randomizado conduzido em 30 contextos de práticas gerais comunitários e 5 centros de cuidados domiciliários, na Holanda, durante 6 semanas de intervenção e 9 meses de seguimento. 0 estudo recrutou 204 pessoas com DPOC, diferenciandoas em 2 grupos: o grupo de controlo (constituído por 101 pessoas) e um grupo de intervenção (composto por 103 pessoas) ${ }^{(9)}$.

No grupo de intervenção, procedeu-se à aplicação da COPD-GRIP a cada participante. Esta consistiu em 3 consultas extra, com uma duração de 30 minutos, com 3 semanas de intervalo. $\mathrm{Na}$ primeira consulta, o enfermeiro abordou as perceções da pessoa com DPOC sobre a doença, recorrendo ao Brief Illness Perception Questtionaire. Posteriormente, na $2^{\mathrm{a}}$ consulta, procedeu-se à discussão sobre a relação entre as perceções da pessoa e os seus comportamentos, sendo esta convidada a elaborar o seu próprio plano de cuidados. Na última consulta, procedeu-se à avaliação das ações, perceções e comportamentos que a pessoa com DPOC alterou. Após a implementação da intervenção, procedeu-se à análise dos resultados através de um modelo linear misto ${ }^{(9)}$.

O primeiro estudo concluiu que houve uma mudança no Questionário Clínico da DPOC, após 9 meses da intervenção. Paralelamente, a intervenção contribuiu para mudanças ao nível das perceções sobre a DPOC, ao nível da qualidade de vida relacionada com a saúde, ao nível das atividades de vida diária e ao nível da educação para a saúde. No grupo de intervenção, verificou-se um significativo aumento no comportamento relacionado com a saúde às 6 semanas $(p=0,024)$ e no controlo pessoal $(p=0,005)$ aos 9 meses. Ainda assim, não se verificou uma mudança significativa entre 0 grupo de controlo e 0 de intervenção, aos 9 meses.

Assim, os autores concluíram que a intervenção DPOCGRIP não melhorou o estado de saúde em pessoas com DPOC, ao nível dos cuidados de saúde primários. No entanto, a intervenção trouxe benefícios ao melhorar a capacidade de controlo da doença e dos 
comportamentos relacionados com a saúde, a curto prazo ${ }^{(9)}$.

O segundo estudo foi desenvolvido em Lincolnshire e pretendeu avaliar a segurança e eficácia de um serviço especializado de avaliação respiratória aguda para pessoas com DPOC, liderado por enfermeiros (Respiratory Nurse Specialist) ${ }^{(10)}$. Os autores analisaram as notas de casos, referentes a pessoas que haviam sido encaminhadas ao serviço, durante um período de 12 meses. Simultaneamente, procederam à aplicação e análise de questionários (numa escala de likert), de forma a avaliar a satisfação com o serviço ${ }^{(10)}$.

No estudo em questão, participaram 128 pessoas com DPOC, com exacerbação aguda e que necessitavam de apoio para prevenir o internamento. Estas foram reencaminhadas para enfermeiros comunitários especialistas em enfermagem respiratória. O serviço especializado contou com uma avaliação respiratória inicial, desenvolvida por uma enfermeira especialista em enfermagem respiratória (histórico médico, medicação, técnica inalatória, exame físico, avaliações objetivas da temperatura, pulso, frequência respiratória, pressão arterial e gasometria arterial). Seguiu-se uma discussão com cada pessoa com DPOC, onde se elaborou um plano personalizado. Os participantes foram monitorizados no domicílio e por telefone (até 14 dias, conforme gravidade e necessidade de suporte) ${ }^{(10)}$.

Os autores concluíram que o especialista em enfermagem respiratória pode servir de articulação e de elemento referenciador das pessoas com DPOC a outros serviços (ex: terapia ocupacional, fisioterapia, assistência social, hospital de dia, programas de cessação tabágica, programas de reabilitação pulmonar) ${ }^{(10)}$. Consequentemente, constataram que as competências destes especialistas foram um contributo significativo no sucesso do serviço, particularmente as capacidades de interpretar os valores das gasometrias e de iniciar administração emergente de oxigénio, conforme necessário.

Os autores verificaram, também, que desde a introdução deste tipo de serviço, a taxa de admissão hospitalar reduziu significativamente. Ainda assim, admitem que é difícil atribuir a redução da admissão somente a este tipo de serviço(10). Por fim, outro ganho adquirido com este serviço foi a educação fornecida sobre a autogestão, uma vez que as pessoas que usufruíram deste tipo de serviço ficaram mais conscientes dos serviços existentes na comunidade. Tal possibilita a elaboração de planos de autogestão adequados, contribuindo para que as pessoas com DPOC mais facilmente procurem tratamento ao primeiro sinal de exacerbação da doença.

A pesquisa atestou, ainda, que os enfermeiros especialistas têm um papel chave ao evitar admissões hospitalares, tendo a capacidade de cuidar de pessoas de forma segura e efetiva e sendo capazes de prescrever tratamentos que, se fossem geridos por enfermeiros generalistas, iriam exigir admissão hospitalar ${ }^{(10)}$.

Segue-se um terceiro estudo, desenvolvido na Coreia, que teve como objetivo avaliar a associação entre o nível de equipas de enfermagem e a taxa de readmissão de pessoas com DPOC ${ }^{(11)}$. Para isso, analisaram os dados nacionais de saúde de 2002 a 2012 e recorreram a uma equação modelar para associar a readmissão aos enfermeiros (Registered Nurses, na Coreia existem Registered Nurses ou Certified Nursing Assistants). Participaram 1070 hospitais e foram estudados 339379 casos de hospitações de pessoas com DPOC. Os autores dividiram o número de enfermeiros por 100 camas e distinguiram-no em três grupos consoante a proporção de enfermeiros: Grupo 1 (baixa), Grupo 2 (moderada), Grupo 3 (elevada). Posteriormente, identificaram as datas da $1^{\text {a }}$ hospitalização e da alta, seguido de verificação da existência de readmissão num período de 30 dias, sendo cada um considerada como nova hospitalização (após $1^{\text {a }}$ alta).

Os autores consideraram as variáveis da pessoa com DPOC (diagnóstico principal, idade, sexo, dificuldade respiratória, comorbilidades, duração da oxigenoterapia, tempo internamento numa unidade cuidados intensivos,) e as características do hospital (estruturais, recursos humanos, tipo de instituição, número de camas) ${ }^{(11)}$.

Neste estudo, os resultados demonstraram notáveis efeitos positivos dos enfermeiros face à pessoa com DPOC. Não obstante, a magnitude deste impacto divergiu consoante o tamanho dos hospitais. Efetivamente verificou-se um aumento de readmissões nos hospitais com reduzidos números de enfermeiros (mais readmissões no grupo 1 do que no grupo 3). Assim, um número superior de enfermeiros por 100 camas foi significativamente associado a taxas de readmissão inferiores (quando comparado com número inferior de enfermeiros) ${ }^{(11)}$.

Apesar do descrito, os autores reconheceram que o estudo apresentou algumas limitações como a não avaliação de outras características da pessoa com DPOC que poderiam afetar a readmissão (escolaridade, estatuto socioeconómico, gravidade da doença, atual tratamento médico, utilização de outros serviços de saúde, visitas domiciliárias,...), a incapacidade de avaliar os efeitos dos médicos especialistas na readmissão, bem como as mudanças nos recursos humanos (como a taxa de rotatividade dos enfermeiros) ${ }^{(11)}$.

Um outro estudo, oriundo da revisão realizada, tinha como objetivo avaliar se uma intervenção de suporte, via telefone, aumentaria o bem-estar da pessoa e reduziria a gravidade dos sintomas, no contexto saúde primário, em Londres. Este estudo tinha como objetivos: determinar a viabilidade do estudo, da intervenção, determinar os efeitos secundários da intervenção e dos seus $\operatorname{custos}^{(12)}$.

Para isso, os autores delinearam um estudo randomizado com 2 grupos (controlo e intervenção) e procederam à aplicação dos questionários Chronic Obstructive Assessment Tool (CAT). Participaram 71 pessoas com DPOC, a viver na comunidade, e acompanhados em contexto cuidados saúde primários (34 pessoas no grupo de intervenção e 35 no grupo de controlo). O grupo de Intervenção recebeu o mesmo tratamento que o grupo de controlo, tendo participado 
numa intervenção de mais 6 semanas. Neste período, as pessoas do grupo de intervenção foram contactadas 2 vezes (na $3^{\mathrm{a}}$ e $5^{\mathrm{a}}$ semana) por um enfermeiro de prática avançada (Advanced Nurse Practitioner). Cada chamada teve cerca de 25 minutos e abordou as seguintes questões: viver com a condição, uso do plano de ação face aos sintomas, uso medicação, encorajamento e a disponibilização de suporte.

Para além disto, o enfermeiro fez uma revisão da informação necessária à autogestão e respondeu a questões sobre o plano de autogestão, gestão de sintomas e início de medicação de emergência. 0 enfermeiro também avaliou a autopercepção da pessoa sobre a sua saúde, e sugeriu visitas clínicas, quando necessário. Paralalemente, o enfermeiro avaliou todos os relatórios médicos, no momento da intervenção telefónica e recorreu a questões abertas, de forma a promover a discussão(12).

De forma a avaliar os resultados, os autores procederam à avaliação do impacto nos sintomas através do CAT (no início e ao fim de 12 semanas), ao número de exacerbações autorreportadas e à satisfação com o serviço. No grupo de intervenção, o score do CAT diminuiu significativamente, demonstrando melhoria entre o momento 1 e o momento 2. No grupo de controlo, não se verificaram mudanças. No score do CAT, verificou-se uma diferença significativa entre os dois grupos, no $2^{\circ}$ momento. Porém, não se verificaram mudanças significativas, no que se refere a exarcebações no segundo momento, bem como no que se refere à satisfação (que não se diferenciou significativamente entre os dois grupos). Os autores concluíram que a intervenção de enfermagem via telefónica é válida, em contexto de cuidados de saúde primários, podendo ajudar a melhorar a saúde e o bemestar das pessoas ${ }^{(12)}$.

Por sua vez, outros pesquisadores desenvolveram um estudo em Victoria, Australia, que tinha como objetivo: avaliar o impacto da introdução de um modelo de cuidados (com enfermeiro de prática avançada em doença crónica respiratória - Chronic Respiratory Disease Nurse Practitioner, ao nível da apreciação da oxigenoterapia de curta duração, na prestação de cuidados e nos resultados para a pessoa com DPOC ${ }^{(11)}$. Procederam a um estudo retrospetivo não controlado, através de auditorias clínicas, que foram conduzidas em 2 momentos (pré e pós introdução do modelo de cuidados, em 2009 e 2011 respetivamente) ${ }^{(13)}$.

Participaram 301 pessoas com DPOC com infeção/exacerbação respiratória aguda, tendo colhido os dados a partir dos relatórios médicos. Os dados colhidos foram: saturação de oxigénio (48h antes da alta), colheita de gasometria arterial (48h antes da alta) nos casos de saturações inferiores ou iguais a $90 \%$, existência ou não de teste de marcha funcional e saturações de oxigénio inferiores ou iguais a $88 \%$ (48h antes da alta), elegibilidade da pessoa para receber oxigenoterapia curta duração, existência de recomendação para oxigenoterapia de curta duração (no momento da alta) e existência de reinternamento, dentro de 28 dias, com um diagnóstico associado à DPOC $^{(13)}$.
Os resultados revelaram a existência de um aumento significativo de pessoas avaliadas com gasometrias arteriais, para avaliar a necessidade de oxigenoterapia de curta duração, de 7,7\% (2009) para 45\% (2011). 0 estudo revelou que a necessidade de oxigenoterpia de curta duração aumentou de $26,7 \%$ para $44,4 \%$ (13).

Paralelamente, os reinternamentos hospitalares, no período de 28 dias pós-alta, em pessoas com oxigenoterapia de curta duração reduziram de 25\% para $12,5 \%$. Assim, os autores concluíram que, com a introdução do modelo de cuidados, houve um aumento do número de pessoas avaliadas para a elegibilidade e a receberem oxigenoterapia de curta duração, uma redução dos reinternamentos hospitalares, uma melhoria dos protocolos de atuação, bem como uma melhoria nos ganhos de saúde e uma poupança financeira para o hospital estudado(13).

Por último, segue-se um estudo realizado em hospitais da Tailândia. Este estudo teve como objetivos explorar o nível de implementação de um modelo de cuidados, num programa de DPOC e comparar a diferença dos resultados das equipas sem e com enfermeiros de prática avançada (Advanced Practice Nurses). Os autores procederam a um estudo comparativo descritivo, através da aplicação de questionários, da avaliação da função respiratória e da realização de um teste piloto (para avaliar a validade dos instrumentos) (14).

Participaram 210 pessoas com DPOC que, 1 ano antes, tiveram uma adesão de $80 \%$ às consultas de DPOC e que fossem capazes de comunicar. Os participantes foram divididos num grupo controlo, que foi cuidado por equipas sem enfermeiros de prática avançada (105 pessoas), e num grupo de intervenção, que foi cuidado por equipas com enfermeiros de prática avançada (105 pessoas) $)^{(14)}$.

Sob a forma de entrevista, os autores aplicaram os questionários na seguinte ordem: Personal Information Questtionnaire, Self-Care Behaviors Questionnaire, Health-Related Quality of Life Questionnaire e Satisfaction with Care Questionnaire. Seguiram com a realização do teste 6 minutos de marcha e de provas função respiratória. Posteriormente, procederam à revisão do número de idas às urgências, das admissões hospitalares, do tempo de internamento e dos custos dos cuidados de saúde (registados nos relatórios médicos), do último ano. Ainda durante o processo, os profissionais das equipas foram convidados a responder em conjunto ao Assessment of Chronic Illness Care ${ }^{(14)}$.

0 estudo revelou que o grupo de intervenção apresentou comportamentos de autocuidado e função pulmonar mais elevados, bem como um tempo de internamento e custos com cuidados de saúde mais baixos. Concomitantemente, os autores verificaram que os enfermeiros de prática avançada podem liderar e apoiar as equipas multidisciplinares, ao educar e ao desenvolver competências, que capacitam as pessoas a desempenharem um autocuidado apropriado (ex: uso preciso dos inaladores, promoção de exercício físico e de respiração adequada, cessação tabágica, reconhecimento precoce e prevenção de exacerbações, ... $)^{(14)}$. 
Além disto, os enfermeiros de prática avançada integraram outros componentes do modelo, ao usarem guidelines clínicas e ao providenciarem um cuidado efetivo (através do planeamento cuidados, consultas, visitas domiciliárias e gestão de casos). Também a articulação dos enfermeiros de prática avançada com os recursos da comunidade e com os restantes membros das equipas possibilitaram a criação de interações produtivas, com o objetivo da melhoria funcional e da melhoria da qualidade de vida da pessoa. Um exemplo positivo foi a ação de uma enfermeira de prática avançada, que desenvolveu uma garrafa de sopro, para assistir as pessoas a realizarem os exercícios respiratórios, facilitando o seu autocuidado, os seus conhecimentos sobre a doença e a função pulmonar. Por fim, o estudo revelou que a intervenção destes enfermeiros, nos programas da DPOC, resultou em ganhos, nomeadamente na melhoria da função pulmonar/comportamentos de autocuidado e na redução de custos com serviços e do tempo internamento $^{(14)}$.

\section{DISCUSSÃO}

Os estudos utilizados evidenciaram uma multiplicidade nas formas de atuação do Enfermeiro Reabilitação nomeadamente o COPD-GRIP, a intervenção telefónica, os modelos de cuidados com Enfermagem respiratória ou a avaliação da necessidade de oxigenoterapia de curta duração. Ainda assim, seja qual for a forma de atuação, notou-se uma preferência pela realização de estudos controlados randomizados (com grupos de intervenção e controlo). Isto vai ao encontro da literatura, onde se destaca a comparação dos resultados entre pessoas submetidas a programas de reabilitação respiratória e pessoas não submetidas aos mesmos $^{(15-16)}$.

Os resultados revelaram concordância sobre o impacto ao nível da redução das (re)admissões hospitalares(10$11,13)$, o aumento da perceção do controlo da doença e dos meios envolventes $(9-11,14)$. Paralelamente, os estudos demonstraram uma relação direta entre a atuação do ER e a redução do tempo de internamento e encargos financeiros para as instituições de saúde ${ }^{(13-}$ 14).

Assim, os resultados obtidos parecem estar consonantes com alguns autores. Efetivamente, num estudo, onde se procurou estimar o custo das exacerbações das pessoas com DPOC, submetidas a Programa de Reabilitação Pulmonar, os autores constataram que uma maior permanência no programa estava diretamente relacionada com redução do número e da gravidade de exacerbações, bem como da redução dos custos ${ }^{(2)}$.

Para além do referido, os resultados corroboram um estudo, onde os autores ao comparar 2 grupos (com e sem intervenção do enfermeiro de reabilitação) concluíram que o grupo que recebeu intervenção do enfermeiro de reabilitação obteve ganhos nas duas grandes dimensões do Questionário de Saúde SF-36, no desempenho físico, saúde geral e vitalidade ${ }^{(15)}$.
Comparando os resultados obtidos com o estado da arte, verificou-se concordância na melhoria da tolerância à atividade e da qualidade de vida. Porém, somente um estudo revelou-se concordante com a melhoria na dispneia ${ }^{(17)}$.

Torna-se importante realçar que, apesar de poucos países apresentarem enfermeiros especialistas em enfermagem de reabilitação (com as mesmas competências específicas que a legislada no contexto português), muitos apresentam enfermeiros avançados na área da doença crónica respiratória. Estes peritos ("enfermeiros respiratórios") apresentam formação, conhecimento e experiência clínica, executando intervenções similares na abordagem à pessoa com DPOC. De facto, os artigos analisados destacam "olhar" especializado dos enfermeiros de reabilitação face à pessoa. Tal vai ao encontro da literatura que reconhece que os enfermeiros de reabilitação reconhecem o impacto do contexto e do ambiente, sendo essenciais para gerir situações complexas e intervir em qualquer etapa da vida, estabelecendo parcerias com as pessoas e as comunidades e coordenando planos de cuidados interdisciplinares ${ }^{(18)}$.

Por fim, salienta-se que os estudos convergem em determinados aspetos sobre os ganhos do Enfermeiro de Reabilitação, refletindo sobre a realidade de alguns contextos. Ainda assim, é de realçar a pouca investigação existente sobre resultados das intervenções.

\section{CONCLUSÃO}

A presente revisão sistemática propunha-se responder à questão norteadora "Qual o impacto da intervenção do Enfermeiro de Reabilitação na Pessoa com DPOC?”, procurando evidenciar os resultados da intervenção do Enfermeiro de Reabilitação. Os estudos destacaram diversidade nas formas de agir do Enfermeiro de Reabilitação. Os resultados foram consonantes no que se refere ao impacto na redução das (re)admissões hospitalares e no aumento de controlo da doença.

Verificou-se uma relação direta entre a atuação do Enfermeiro de Reabilitação e a redução do tempo de internamento e dos custos para as organizações, o que pode ter implicações para as tomadas de decisão dos gestores de saúde. Além disto, os estudos convergem em alguns aspetos sobre os ganhos do Enfermeiro de Reabilitação, respondendo à pergunta inicial e refletindo sobre a realidade de alguns contextos de saúde.

Esta revisão tem, pois, implicações ao nível do ensino, uma vez que demonstra aos alunos, a importância de destacar cuidados de enfermagem efetivos. Por fim, sugere-se que se desenvolvam investigações sobre o impacto do Enfermeiro de Reabilitação na adesão ao regime terapêutico (ex: inaloterapia), na pessoa com DPOC.

\section{Limitações da revisão sistemática da literatura}

A presente revisão sistemática apresenta algumas limitações. A primeira prende-se com a limitação dos 
artigos à língua inglesa ou portuguesa, pois esta condiciona 0 aparecimento de artigos publicados noutras línguas. Uma outra limitação é o facto de se terem excluído artigos por não terem texto completo ou por exigirem subscrição no periódico em causa, diminuindo o número de artigos disponíveis. Por fim, a terceira limitação deve-se ao facto de se ter optado por estudar o impacto geral da intervenção do Enfermeiro de Reabilitação, o que poderá ter excluído evidência interessante sobre o impacto económico para a instituição de saúde, para a satisfação da pessoa com DPOC, ou sobre intervenções específicas do Enfermeiro de Reabilitação. Contudo, é importante realçar que o objetivo da presente revisão sistemática nunca foi o de estudar resultados de intervenções específicas do Enfermeiro de Reabilitação, mas sim o de compreender e aprofundar o impacto que a sua abordagem e o seu olhar podem ter face à pessoa com DPOC.

\section{REFERÊNCIAS BIBLIOGRÁFICAS}

1. Global Initiative for chronic Obstructive Lung Disease. Pocket Guide to COPD Diagnosis, Management, and Prevention - A Guide for Health Care Porfessionals. USA: GOLD; 2019

2. Garske CCDG, Scheneiders PB, Silva ALG, Carvalho LL. Custo de exacerbações em pacientes com doença pulmonar obstrutiva crônica submetidos a um programa de reabilitação pulmonar. R Epidemiol Control Infec. 2018; $8^{(3)}$ : 204-209.

3. Santos AC, Barreto C, Barata F, Froes F, Carvalho I, Pité I, et al. $13^{\circ}$ Relatório do Observatório Nacional das Doenças Respiratórias 2016/2017 - Panorama das Doenças Respiratórias em Portugal - Retrato da saúde 2048. Lisboa: Observatório Nacional das Doenças Respiratórias; 2018.

4. Direção-Geral de Saúde. Programa Nacional para as Doenças Respiratórias. Lisboa: Ministério da Saúde; 2017.

5. 5 Direção-Geral da Saúde. Circular Informativa $n^{\circ} 40^{a} / D S P C D$ de 27 de outubro de 2009. Orientações Técnicas sobre Reabilitação Respiratória na Doença Pulmonar Obstrutiva Crónica (DPOC); 2009.

6. Cordeiro MCO, Menoita ECPC. Manual de Boas Práticas na Reabilitação Respiratória - Conceitos, Princípios e Técnicas.

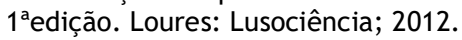

7. Assembleia do Colégio da Especialidade de Enfermagem de Reabilitação. Áreas de Investigação Prioritárias para a Especialidade de Enfermagem de Reabilitação. Porto: Ordem dos Enfermeiros; 2015

8. The Joanna Briggs Institute. Joanna Briggs Institute Reviewers'Manual: 2011 edition. Adelaide: The Joanna Briggs Institute; 2011

9. Weldam SW, Schuurmans MJ, Zanen P, Heijmans MJ, Sachs AP, Lammers JWJ. The effectiveness of a nurse-led illness perception intervention in COPD patients: a cluster randomised trial in primary care. ERJ Open Res. 2017; 3: 1-13.

10. Cox K, Macleod SC, Sim CJ, Jones AW, Trueman J. Avoiding hospital admission in COPD impact of a specialist nursing team. Br J Nurs. 2017; 26 ${ }^{(3)}$ : 152-158.

11. Kim J, Park EC, Han KT, Kim SJ, Kim TH. Nurse Staffing and 30day Readmission of Chronic Obstructive Pulmonary Disease Patients: A 10-year Retrospective Study of Patient Hospitalization. Asian Nurs Res. 2016; 10: 283-286.

12. Bilington J, Coster S, Murrells T, Norman I. Evaluation of a NurseLed Educational Telephone Intervention to Support SelfManagement of Patients With Chronic Obstructive Pulmonary Disease: A Randomized Feasibility Study. COPD. 2015; 12: 395 403.

13. Champion R, Hall T, Tori K. Home oxygen therapy assessment for COPD patients discharged from hospital: Respiratory NP Model of Care. Aust J Adv Nurs. 2015; 33 ${ }^{(4)}: 17-25$.

14. Duangubpha S, Hanucharurnkul S, Pookboonmee R, Orathai P, Kiatboonri C. Chronic Care Model Implementation and Outcomes among Patients with COPD in Care Teams with and without
Advanced Practice Nurses. Pac Rim Int J Nurs Res Thail. 2013 April-June; $17^{(2)}: 102-115$.

15. Fernando JAC. Ganhos em saúde com a intervenção dos enfermeiros de reabilitação em doentes com DPOC. Vila Nova de Gaia. Dissertação [Mestre em Gestão das Organizações, Ramo de Gestão de Unidades de Saúde] - Instituto Politécnico de Bragança; 2016.

16. Gi MMFP. Doença Pulmonar Obstruttiva Crónica, Identificação de Fenómenos Específicos de Intervenção do Enfermeiro Especialista de Reabilitação [Dissertação]. Bragança: Instituto Politécnico de Bragança; 2012.

17. Gomes MS. A implementação de programas de reabilitação respiratória em pessoas com DPOC : uma revisão sistemática da literatura [Dissertação]. Porto: Escola Superior de Enfermagem do Porto; 2016.

18. Hoeman SP. Enfermagem de Reabilitação - Prevenção, Intervenção e Resultados Esperados. $4^{a}$ edição. Loures: Lusociência, 2011 